\title{
A method for the design of unsymmetrical optical systems using freeform surfaces
}

Dmitry Reshidko, Jose Sasian

Dmitry Reshidko, Jose Sasian, "A method for the design of unsymmetrical optical systems using freeform surfaces," Proc. SPIE 10590, International Optical Design Conference 2017, 105900V (27 November 2017); doi: $10.1117 / 12.2285134$

SPIE Event: International Optical Design Conference - IODC 2017, 2017, Denver, United States 


\title{
A method for the design of unsymmetrical optical systems using freeform surfaces
}

\author{
Dmitry Reshidko*, Jose Sasian \\ University of Arizona, College of Optical Sciences, 1630 E University Blvd., Tucson, USA, 85721
}

\begin{abstract}
A systematic method for the design of unsymmetrical optical systems is described. Freeform optical surfaces are constructed by superposition of a conic segment and a polynomial, and successfully applied to design relatively fast wide field-of-view optical systems.
\end{abstract}

Keywords: Unsymmetrical optical systems, freeform surfaces, optical design, aberration correction

\section{INTRODUCTION}

Optical systems that do not have axial symmetry can provide useful and unique solutions to certain imaging problems. However, the complexity of the optical design task grows as the degrees of symmetry are reduced and lost: there are more aberration terms to control, and achieving a sharp image over a wide field-of-view at fast optical speeds becomes challenging. Plane-symmetric optical systems represent a large family of practical non-axially symmetric systems that are simple enough to be easily described and thus are well understood. Design methodologies and aberration theory of plane-symmetric optical systems have been discussed in the literature, and various interesting solutions have been reported [1-4].

The little discussed in the literature technique of confocal systems is effective for the design of unsymmetrical optics. A confocal unsymmetrical system is constructed in such a way that there is sharp image along a given ray (called the optical axis ray (OAR)) surface after surface. It is possible to show that such a system can have a reduced number of field aberrations, and that the system will behave closer to an axially symmetric system [5-6].

In this paper, we review a methodology for the design of unsymmetrical optical systems. We utilize an aspherical/freeform surface constructed by superposition of a conic expressed in a coordinate system that is centered on the off-axis surface segment rather than centered on the axis of symmetry, and an XY polynomial. The conic part of the aspherical/freeform surface describes the base shape that is required to achieve stigmatic imaging surface after surface along the OAR. The XY polynomial adds a more refined shape description to the surface sag and provides effective degrees of freedom for higher-order aberration correction. This aspheric/freeform surface profile is able to best model the ideal reflective surface and to allow one to intelligently approach the optical design. Examples of two- and threemirror unobscured wide field-of-view reflective systems are provided to show how the methods and corresponding aspheric/freeform surface are applied. We also demonstrate how the method can be extended to design a monolithic freeform objective.

\section{ABERRATIONS OF PLANE-SYMMETRIC OPTICAL SYSTEMS}

In this section, we review the aberration properties of a plane-symmetric optical system. The well-known concept of the axially symmetric wavefront aberration function $W(\vec{H}, \vec{\rho})$ is extended to describe the imaginary of plane-symmetric systems. The wavefront aberration function $W(\vec{H}, \vec{\rho}, \vec{i})$ of a plane-symmetric optical system gives the geometrical wavefront deformation at the exit pupil as a function of the normalized field $\vec{H}$, aperture $\vec{\rho}$, and symmetry unit $\vec{i}$ vectors. The plane of symmetry contains a ray, called the optical axis ray (OAR) which defines the center of the field-ofview (FOV) and the center of the pupils. The unit symmetry vector $\vec{i}$ defines the direction of the plane of symmetry. For optical systems that are composed from spherical or slightly aspherical surfaces, the wavefront aberration function is expanded into polynomial series of dot products of the field, aperture, and symmetry unit vectors, and can be written as

International Optical Design Conference 2017, edited by Peter P. Clark, Julius A. Muschaweck,

Richard N. Pfisterer, John R. Rogers, Proc. of SPIE-OSA Vol. 10590, 105900V · @ 2017 SPIE

CCC code: $0277-786 X / 17 / \$ 18 \cdot$ doi: $10.1117 / 12.2285134$ 


$$
W(\vec{H}, \vec{\rho}, \vec{i})=\sum_{k, m, n, p, q} W_{\substack{2 k+n+p, 2 m+n, q \\ n, p, q}}(\vec{H} \cdot \vec{H})^{k}(\vec{\rho} \cdot \vec{\rho})^{m}(\vec{H} \cdot \vec{\rho})^{n}(\vec{H} \cdot \vec{i})^{p}(\vec{\rho} \cdot \vec{i})^{q},
$$

where each aberration coefficient $W_{2 k+n+p, 2 m+n+q, n, p, q}$ represents the amplitude of basic wavefront deformation forms defined by the integers $k, m, n, p$, and $q$. The sum of these integers represents a certain order of approximation.

The aberration terms to the fourth order of approximation are summarized in Table 1. These terms consist of the wellunderstood axially-symmetric primary aberrations and an additional set of aberrations that have double-plane and plane symmetry. The magnitude of the aberration coefficients can be calculated from a first-order ray trace and the system structure parameters [1].

Table 1. Aberrations of a plane-symmetric optical system. The aberration terms are arranged in groups according to symmetry characteristics.

\begin{tabular}{|c|c|c|}
\hline \multicolumn{3}{|l|}{ First Group } \\
\hline \multicolumn{3}{|l|}{ Second Group } \\
\hline & $W_{01001}(\vec{i} \cdot \vec{\rho})$ & Field Displacement \\
\hline & $W_{10010}(\vec{i} \cdot \vec{H})$ & Linear Piston \\
\hline & $W_{02000}(\vec{\rho} \cdot \vec{\rho})$ & Defocus \\
\hline & $W_{11100}(\vec{H} \cdot \vec{\rho})$ & Magnification \\
\hline & $W_{20000}(\vec{H} \cdot \vec{H})$ & Quadratic Piston \\
\hline \multicolumn{3}{|l|}{ Third Group } \\
\hline & $W_{02002}(\vec{i} \cdot \vec{\rho})^{2}$ & Uniform Astigmatism \\
\hline & $W_{11011}(\vec{i} \cdot \vec{H})(\vec{i} \cdot \vec{\rho})$ & Anamorphic Distortion \\
\hline & $W_{20020}(\vec{i} \cdot \vec{H})^{2}$ & Quadratic Piston \\
\hline & $W_{03001}(\vec{i} \cdot \vec{\rho})(\vec{\rho} \cdot \vec{\rho})$ & Uniform Coma \\
\hline & $W_{12101}(\vec{i} \cdot \vec{\rho})(\vec{H} \cdot \vec{\rho})$ & Linear Astigmatism \\
\hline & $W_{12010}(\vec{i} \cdot \vec{H})(\vec{\rho} \cdot \vec{\rho})$ & Field Tilt \\
\hline & $W_{21001}(\vec{i} \cdot \vec{\rho})(\vec{H} \cdot \vec{H})$ & Quadratic Distortion I \\
\hline & $W_{21110}(\vec{i} \cdot \vec{H})(\vec{H} \cdot \vec{\rho})$ & Quadratic Distortion II \\
\hline & $W_{30010}(\vec{i} \cdot \vec{H})(\vec{H} \cdot \vec{H})$ & Cubic Piston \\
\hline & $W_{04000}(\vec{\rho} \cdot \vec{\rho})^{2}$ & Spherical Aberration \\
\hline & $W_{13100}(\vec{H} \cdot \vec{\rho})(\vec{\rho} \cdot \vec{\rho})$ & Linear Coma \\
\hline & $W_{22200}(\vec{H} \cdot \vec{\rho})^{2}$ & Quadratic Astigmatism \\
\hline & $W_{22000}(\vec{H} \cdot \vec{H})(\vec{\rho} \cdot \vec{\rho})$ & Field Curvature \\
\hline & $W_{31100}(\vec{H} \cdot \vec{H})(\vec{H} \cdot \vec{\rho})$ & Cubic Distortion \\
\hline & $W_{40000}(\vec{H} \cdot \vec{H})^{2}$ & Quadratic Piston \\
\hline
\end{tabular}

The aberration formulas provide insight into the aberration dependence as a function of the system parameters. Moreover, the aberration division into subgroups according to symmetry characteristics reveals the design strategy and indicates the effective degrees of freedom during the optimization process. 
An important case to highlight is a plane-symmetric optical system constructed with confocal surfaces such as imaging along the OAR is stigmatic surface after surface. This type of systems has a reduced number of aberrations and potentially can provide better imaging. To satisfy the requirement in the case of reflective systems, surfaces must be offaxis segments of conics. Due to the system construction, there will be no field independent aberration terms: the surface contributions to spherical aberration $\left(W_{04000}\right)$, uniform coma $\left(W_{03001}\right)$, and uniform astigmatism $\left(W_{02002}\right)$ are all exactly zero. In addition, anamorphic distortion $\left(W_{11011}\right)$ and quadratic distortion II $\left(W_{21110}\right)$ also nullify, and the surface contribution to intrinsic anamorphism becomes unity. Moreover, linear astigmatism $\left(W_{12101}\right)$ and field tilt $\left(W_{12010}\right)$ of the system vanish simultaneously and can be corrected by adjusting the tilt of mirrors. It follows that except for the remaining quadratic distortion I and to the fourth order of approximation, the system behaves as an axially symmetric system.

\section{SURFACE DESCRIPTION}

In this section, we present an aspherical/freeform surface in close form that can be used to design plane-symmetric optical systems. We have shown that concatenation of stigmatic components is useful to obtain a starting point for designing a system. A standard tilted and/or decentered axially-symmetric conic surface of Eq. 2 provides solution to the problem

$$
z(r)=\frac{c r^{2}}{1+\sqrt{1-(1+k) c^{2} r^{2}}},
$$

where $c$ is the curvature of the surface, $k$ is the conic constant, and $r=\sqrt{x^{2}+y^{2}}$. However, when one wishes to design a plane-symmetric optical system, it is desirable to have a convenient expression for the surface in a coordinate system that is centered on the off-axis surface segment rather that centered on the axis of symmetry. Some advantages of using this surface description are that the actual surface can be precisely specified, that the system geometry can be easily established and optimized in lens design software, and that additional aspheric terms can be added to provide effective degrees of freedom to further improve the system.

The derivation of the analytical expression of the conic as viewed from a coordinate system that is tangent to the surface at a general point away from the axis of symmetry is reviewed below [7, 8]. Original coordinates $(y, z)$ and new coordinates $\left(y^{\prime}, z^{\prime}\right)$ are shown in Fig. 1. Without loss of generality, the new coordinate origin is chosen on the $y$ axis; thus, $Y_{0}$ is the distance from the rotation axis to the new coordinate center.

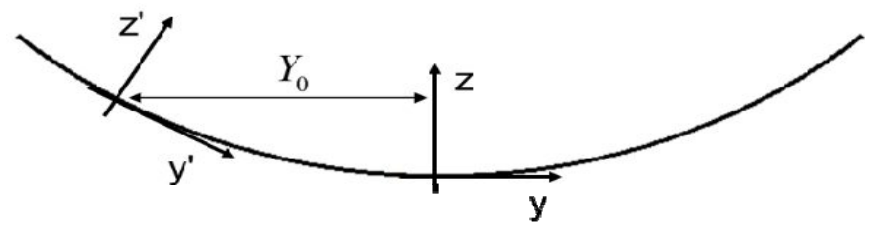

Figure 1. The geometry defining global and local coordinates of the off-axis conic segment.

A general expression of an axially symmetric conic surface of Eq. 2 is rewritten as

$$
z(r)=\frac{1}{(1+k)}\left\{R-\left[R^{2}-(1+k) \cdot r^{2}\right]^{1 / 2}\right\},
$$

where $R=1 / c$ is the radius of curvature of the surface. From Eq. 3, it follows that

$$
\begin{gathered}
\tan \left(\varphi_{0}\right)=\frac{\partial z}{\partial y_{x=0 ; y=Y_{0}}}=\frac{Y_{0}}{\left[R^{2}-(1+k) \cdot Y_{0}^{2}\right]^{1 / 2}}, \\
Z_{0}=z\left(0, Y_{0}\right)=\frac{1}{(1+k)}\left\{R-\left[R^{2}-(1+k) \cdot Y_{0}^{2}\right]^{1 / 2}\right\},
\end{gathered}
$$


where $\varphi_{0}$ is the angle of the coordinate system rotation.

The rotation of coordinates is now performed according to

$$
\begin{gathered}
x=x^{\prime}, \\
y=y^{\prime} \cos \left(\varphi_{0}\right)-z^{\prime} \cdot \sin \left(\varphi_{0}\right)+Y_{0}, \\
z=y^{\prime} \cdot \sin \left(\varphi_{0}\right)+z^{\prime} \cdot \cos \left(\varphi_{0}\right)+Z_{0}, \\
x^{\prime}=x, \\
y^{\prime}=\left(\mathrm{y}-Y_{0}\right) \cdot \cos \left(\varphi_{0}\right)+\left(z^{\prime}-Z_{0}\right) \cdot \sin \left(\varphi_{0}\right), \\
z^{\prime}=-\left(\mathrm{y}-Y_{0}\right) \cdot \sin \left(\varphi_{0}\right)+\left(z^{\prime}-Z_{0}\right) \cdot \cos \left(\varphi_{0}\right) .
\end{gathered}
$$

For compactness, the dimensionless variables

$$
u=\frac{x^{\prime}}{R} ; v=\frac{\mathrm{y}^{\prime}}{R} ; w=\frac{z^{\prime}}{R} ; \varepsilon=\frac{Y_{0}}{R} ;
$$

and the quantities

$$
\begin{aligned}
& s \equiv \sin \left(\varphi_{0}\right)=\frac{Y_{0}}{\left[R^{2}-k Y_{0}^{2}\right]^{1 / 2}}, \\
& c \equiv \cos \left(\varphi_{0}\right)=\left[\frac{R^{2}-(k+1) Y_{0}^{2}}{R^{2}-k Y_{0}^{2}}\right]^{1 / 2}, \\
& L \equiv k+1, \\
& W_{0} \equiv Z_{0} / R
\end{aligned}
$$

are introduced. Eq. 5.1, Eq. 5.2, Eq. 3, Eq. 7, and Eq. 8 are now substituted into Eq. 5.3

$$
\left.\frac{1}{L}\left(1-L\left([v c-w s+\varepsilon]^{2}+u^{2}\right]^{1 / 2}\right)\right)=v s+w c+W_{0} .
$$

After some algebraic manipulations, Eq. 9 is reduced to a quadratic equation as in

$$
w^{2}+2 w(h+j v)-\left(f v^{2}+g u^{2}\right)=0 .
$$

The solution for $w(u, v)$ is

$$
w(u, v)=-(h+j v) \pm\left[(h+j v)^{2}+f v^{2}+g u^{2}\right]^{1 / 2}
$$

where $f \equiv\left(s / \varepsilon^{2}\right)^{2}, g \equiv-1 /\left(L c^{2}+s^{2}\right), h \equiv(\varepsilon / s) g$ and $j \equiv-(L-1) s c g$.

This off-axis conic surface is now used as a base surface to construct an aspherical/freeform surface. The aspherical/freeform surface $z_{f}(r)$ is constructed by adding a plane-symmetric XY polynomial to the base surface as

$$
z_{f}(r)=w(r)+A_{1} x^{2}+A_{2} y^{2}+A_{3} x^{2} y+A_{4} y^{3}+A_{5} x^{4}+A_{6} x^{2} y^{2}+A_{7} y^{4} \ldots,
$$

where $w(r)$ is the sag of the base off-axis conic surface, and $A$ 's are the aspheric polynomial coefficients. The XY polynomial in Eq. 12 is centered at the origin of the off-axis conic segment and thus provides effective degrees of freedom for the correction of off-axis aberrations. This very ad hoc surface allows for optimum design or to refine the design performance of plane-symmetric refractive systems. 


\section{DESIGN METHOD}

In this section, we outline a systematic method to the design of plane-symmetric reflective systems that utilizes the aspheric/freeform surface defined in Section 3:

1. A well-corrected axially symmetric system provides an initial estimate for the first-order parameters and serves as a good starting point for an unsymmetrical design. All surfaces are axially-symmetric conics and imaging is stigmatic along the optical axis surface after surface.

2. Axially-symmetric conic surfaces are converted into aspheric/freeform surfaces. A plane-symmetric form is generated by tilting the system elements in a plane and adding the off-axis conic decenter parameter $Y_{0}$ (see Fig. 1) into optimization. Optimization variables are the surface tilts and separations, and parameter $Y_{0}$. Structure constrains are assigned, and the system is reoptimized to obtain stigmatic imaging along the OAR.

3. Several points in the FOV of the system are added, and the tilt of the last mirror is adjusted to correct linear astigmatism.

4. At this point, the base conic surface is frozen, and only aspheric polynomial coefficients are used by the optimizer as degrees of freedom to correct off-axis aberrations. The coefficients are released as variables and as the optimizer proceeds, more coefficients are released.

Usually, there are several ways to set up a decentered or tilted element in lens design software. We choose a way that is simple to understand, allows to easily construct a system with confocal surfaces, and permits a minimum number of tilt or decenter parameters.

In software, when we tilt and/or decenter a surface, we are actually tilting and/or decentering the local coordinate system in which the surface is defined. Each aspheric/freeform surface is tilted, and the original coordinate system is restored before translating to the next surface. As a result, only the current surface is tilted. A chief ray height solve is assigned to decenter and align the reflected OAR with the vertex of the next surface. Aligning the OAR with the surface vertex of each aspherical/freeform surface allows to effectively control high-order aberrations and to achieve balance performance over the entire field by introducing small asphericities as effective degrees of freedom in step 4.

The surface $z_{f}(r)$ in Eq. 12 was programmed as a user-defined surface in Zemax OpticStudio optical design software and was successfully applied to design several plane-symmetric optical systems. In the following sections, we will present design examples of two- and three-mirror unobscured telescopes. Finally, we will extend our design method to refractive optical systems and show an example of a monolithic freeform objective.

\section{TWO-MIRROR UNOBSCURED TELESCOPE}

In this section, we show a step-by-step design process of a two-mirror unobscured Schwarzschild-type telescope using the systematic method outlined in Section 3. We start with an axially symmetric system shown in Fig. 2a. In this design, curvatures and conic constants of the mirrors are chosen such that imaging on axis is stigmatic surface after surface. The primary mirror is parabolic, and the secondary mirror is hyperbolic. The system operates at $f / 4$. The optical path difference (OPD) plots for 0 and $+/-2$ degrees fields are shown in Fig. $2 b$. The off-axis performance is considerably degraded by coma, field curvature and astigmatism. 


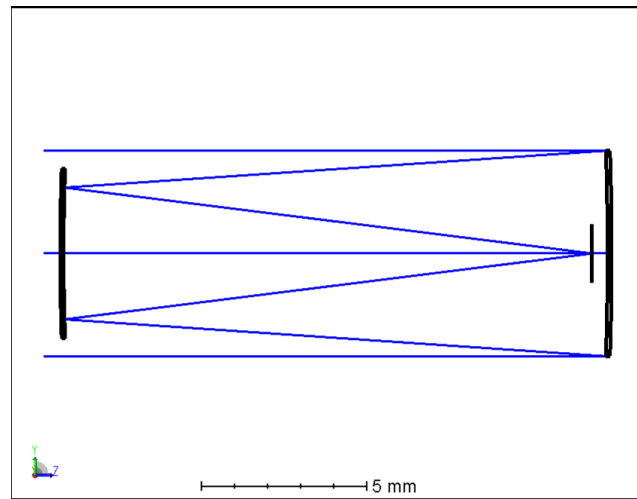

(a)
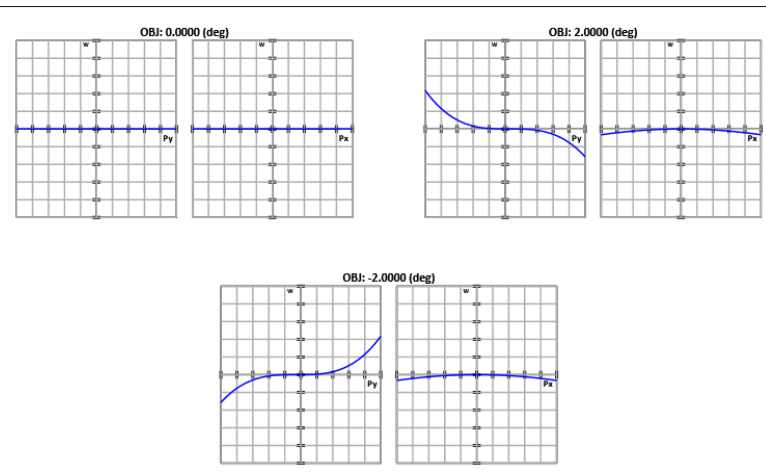

(b)

Figure 2. The starting design of a two-mirror unobscured Schwarzschild-type telescope. (a) Layout; (b) OPD plots (plot scale is 2 waves at $0.550 \mu \mathrm{m}$ ). In this design, imaging on-axis is stigmatic surface after surface.

A plane-symmetric form can be generated by simply tilting and decentering the system elements in a plane. However, if surfaces are defined as standard axially symmetric conic surfaces, only a small off-axis segment of the actual surface is used to bend rays as shown in Fig. 3a. Notice that in this case aspherical polynomial defined with respect to the surface vertex does not provide effective degrees of freedom for further optimization, since higher order polynomial terms are required to produce small aspheric departures at the surface off-axis segment. On the other hand, optical systems that contain lens elements that use higher order aspheric terms are subject to produce oscillation on the ray behavior and are susceptible to creating imaging artifacts when they are slightly misaligned.

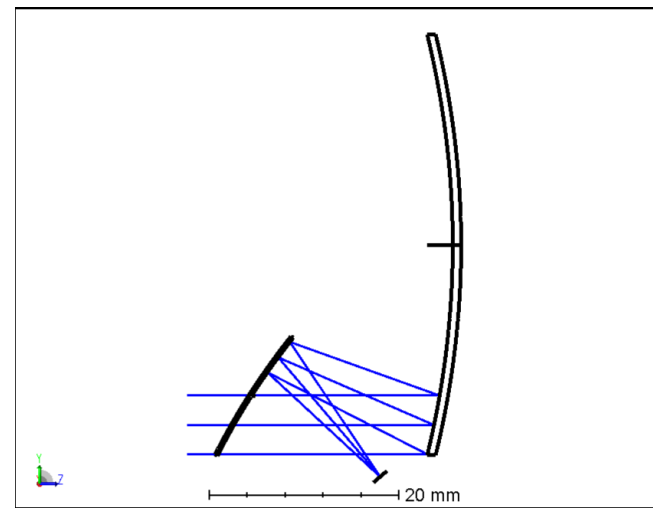

(a)

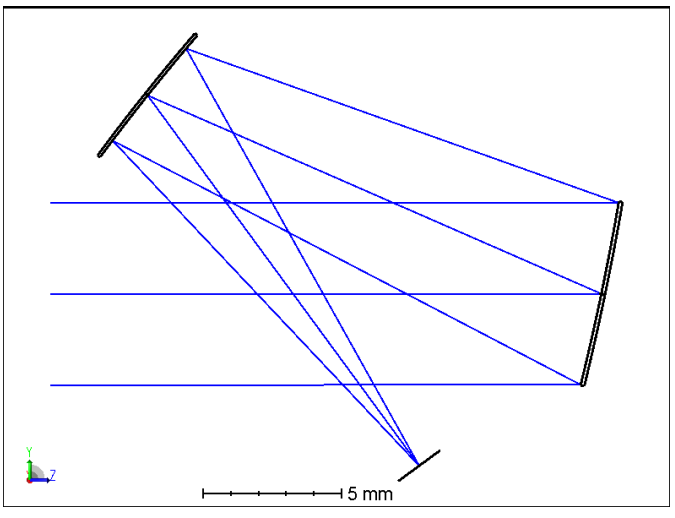

(b)

Figure 3. The elements are tilted and/or decentered to generate an unobscured optical system. Mirror surfaces are defined with (a) standard axially symmetric conic surfaces; (b) aspheric/freeform surfaces $z_{f}(r)$.

Instead, axially-symmetric conic surfaces are converted into aspheric/freeform surfaces $z_{f}(r)$. This surface description simplifies the design by allowing one to set the offset parameter $Y_{0}$ for a given mirror so that stigmatic imaging along the OAR is obtained. The optimization variables are the surface tilts (angle of incidence of the OAR) and surface separations along the OAR, and the parameter $Y_{0}$. The image plane is constrained to be perpendicular to the OAR, and as the system changes it is reoptimized to correct aberrations on axis. The layout is shown in Fig. 3b. The OPD plots of this planesymmetric system are shown in Fig. 4. Notice that due to the system construction, there is no field independent aberration terms. Once the tilt of the secondary mirror is adjusted to correct linear astigmatism and field tilt, the system performs close to the corresponding axially symmetric system. 


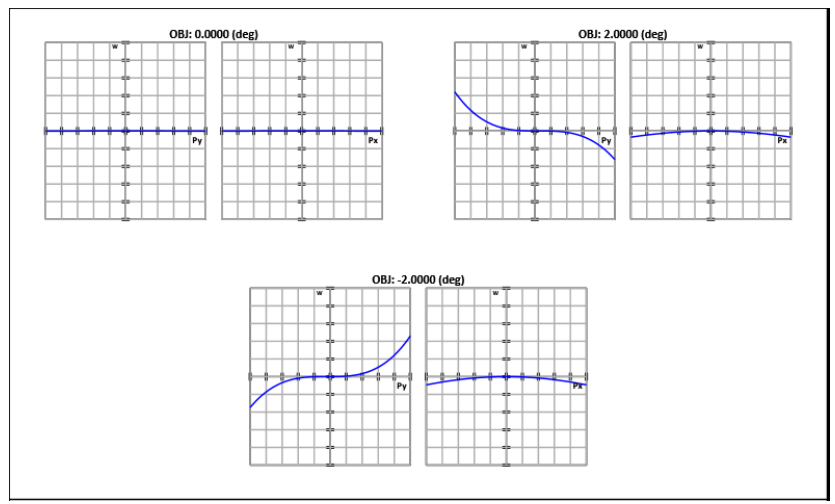

Figure 4. OPD plots of the unobscured telescope (plot scale is 2 waves at $0.550 \mu m$ ). The plane-symmetric system designed with confocal surfaces performs close to the corresponding axially symmetric system.

Off-axis aberrations are now corrected by adding aspheric polynomial coefficients to the optimization. System geometry, surface curvatures and conic constants are locked, and aspheric coefficients are released as variables. The FOV is gradually increased, and more coefficients are released as the optimizer proceeds. The final system, presented in Fig. 5, covers a FOV of $6 \times 4$ degrees at $\mathrm{f} / 4$. The OPD plots and full field spot diagram are shown in Fig. 6. Uniform image quality and close to diffraction limited performance have been achieved over the entire FOV. For reference, two-mirror unobscured telescopes designed with standard conic and aspheric surfaces provide diffraction limited performance over a FOV of only few degrees [3].

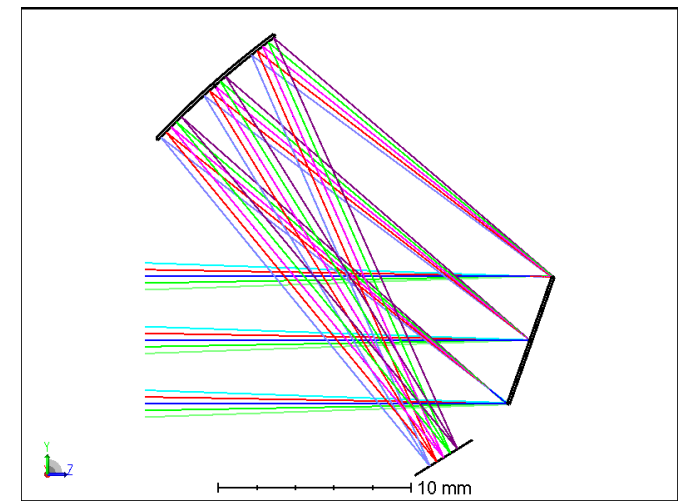

Figure 5. Layout of the final two-mirror unobscured Schwarzschild-type telescope design. Aspheric polynomial terms are used to correct off-axis aberrations.

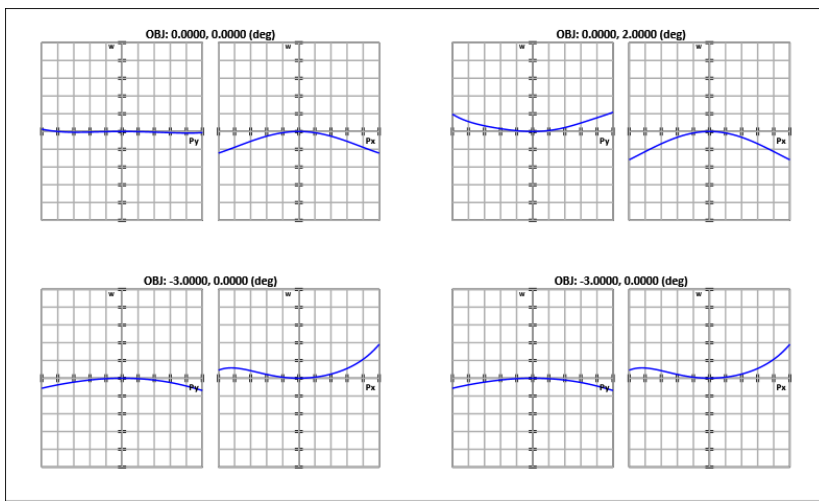

(a)

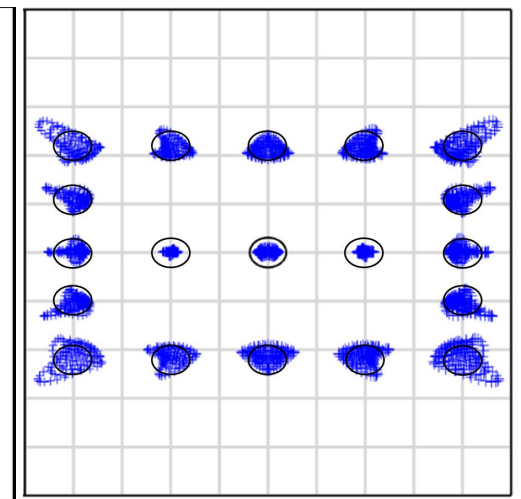

(b)

Figure 6. Imaging performance of the final two-mirror unobscured Schwarzschild-type telescope design. (a) OPD plots (plot scale is 1 wave at $0.550 \mu \mathrm{m}$ ). (b) Full field spot diagram at $0.550 \mu \mathrm{m}$. Close to diffraction limited performance over a FOV of $6 \times 4$ degrees was achieved. 


\section{THREE-MIRROR UNOBSCURED TELESCOPE}

In this section, we present an $\mathrm{f} / 2$ three-mirror unobscured telescope design that closely resembles the non-uniform rational-basis spline (NURBS) freeform design reported by Chrisp and show the performance improvement made by using aspheric surfaces $z_{f}(r)$ [9]. The design parameters are given in Table 2.

Table 2. Design requirements for the three-mirror unobscured system.

\begin{tabular}{lc}
\hline \hline Parameter & Requirement \\
\hline \hline Field of view (FOV) & $10 \times 9$ degrees \\
Focal length & $35.7 \mathrm{~mm}$ \\
Focal ratio & $\mathrm{f} / 2$ \\
\hline \hline
\end{tabular}

The design procedure is similar to one used in the previous section. The initial system is constructed with confocal surfaces, as presentenced in Fig. 7a. Mirror sizes, separations and incidence angles were chosen to closely match the NURBS design by Crisp. Notice that linear astigmatism and field tilt are removed by adjusting the tilt of the tertiary mirror, as shown in Fig $7 \mathrm{~b}$.

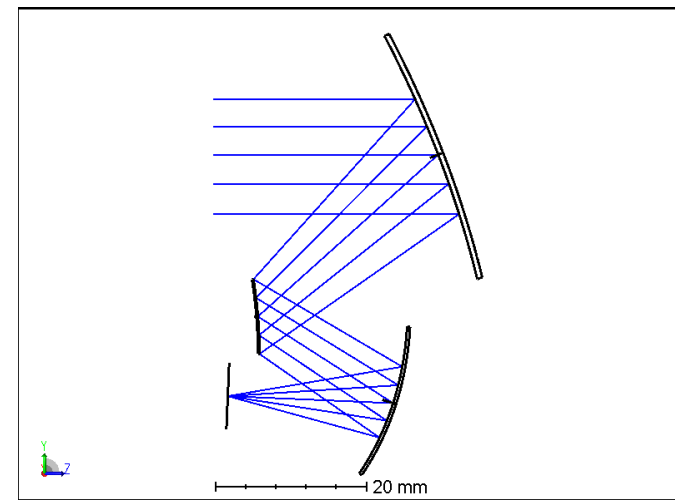

(a)
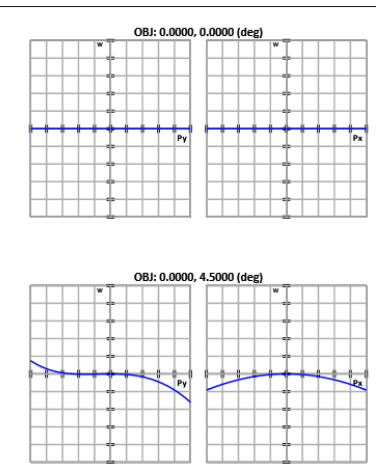
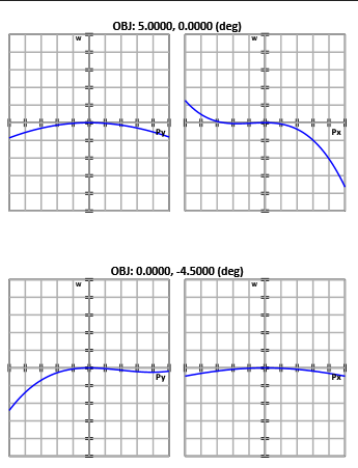

(b)

Figure 7. The starting design of a three-mirror telescope is constructed with confocal surfaces. (a) Layout (b) OPD plots (the plot scale is 10 waves at $3 \mu \mathrm{m}$ ). In this design, imaging on-axis is stigmatic surface after surface. Tilt of the tertiary mirror is adjusted to correct field tilt and linear astigmatism.

Next, mirror curvatures, conic constants, and surface separations are removed from the optimization, and up to $8^{\text {th }}$ order plane-symmetric polynomial coefficients are added as variables to correct off-axis aberrations. Although no constrains on distortion were mentioned by Chrisp, in the current design the distortion is limited to $<3 \%$. The system layout reproduced from the paper by Chrisp and our final system layout are presented side-by-side in Fig. 8. The OPD plots and spot diagrams of our system are given in Fig. 9. The performance is close to being diffraction-limited over the entire FOV. 


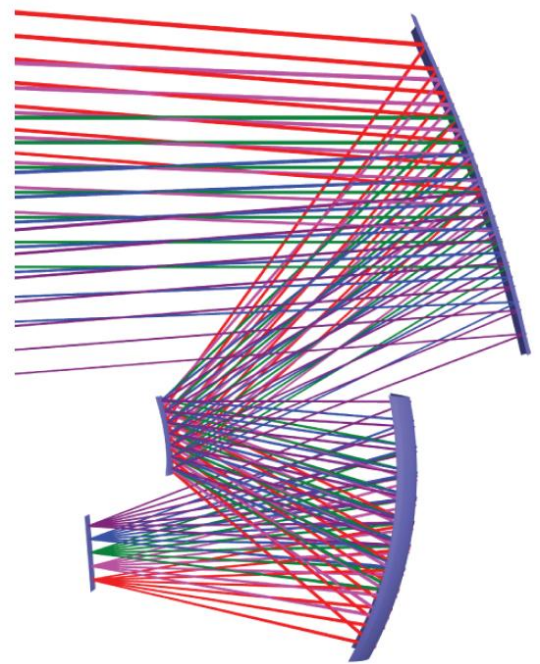

(a)

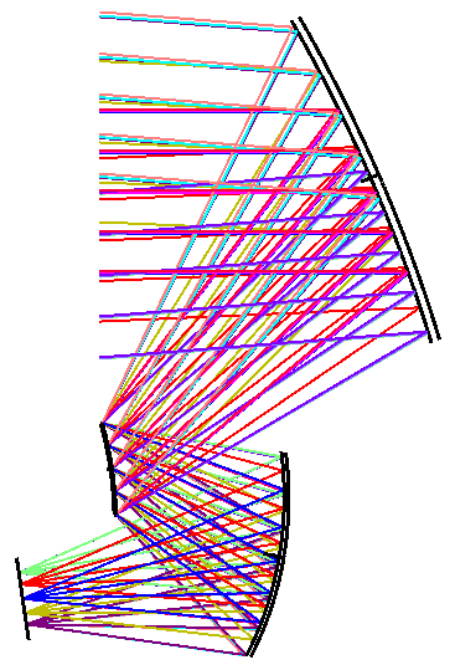

(b)

Figure 8. Layout of the three-mirror telescope. (a) The design with NURBS freeform surfaces reported by Chrisp; (b) the current design with aspherical/freeform surfaces $z_{f}(r)$. In the current design, up to $8^{\text {th }}$ order plane-symmetric polynomials are used to correct off-axis aberrations.

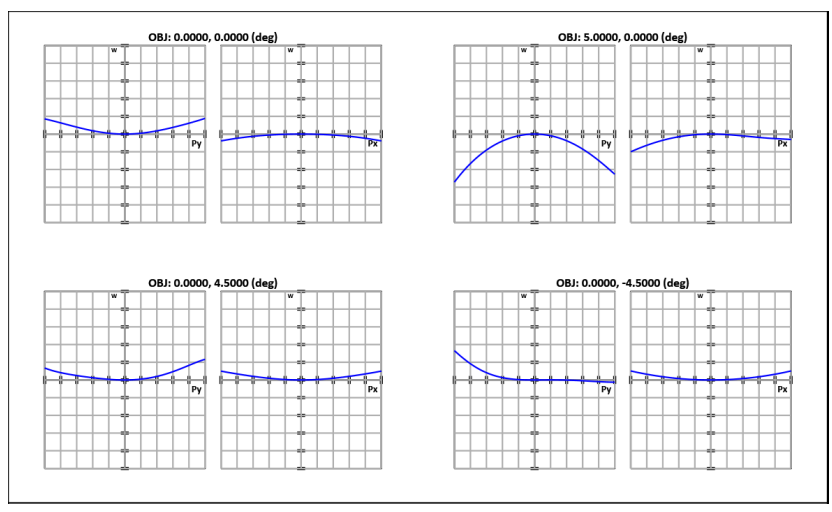

(a)

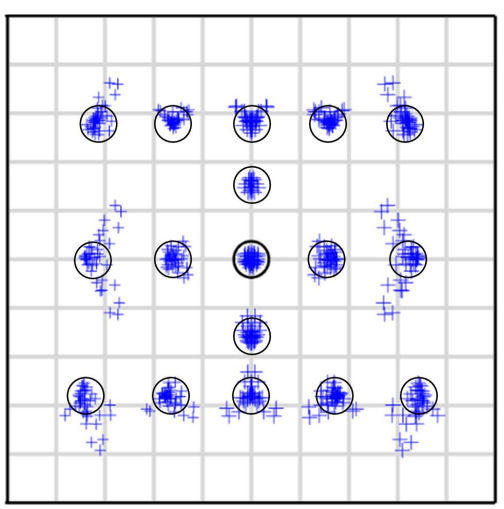

(b)

Figure 9. Imaging performance of the final three-mirror telescope. (a) OPD plots (the plot scale is 1 wave at $3 \mu \mathrm{m}$ ); (b) Spot diagrams at $3 \mu \mathrm{m}$. Close to diffraction limited performance over a FOV of 10x9 degrees was achieved.

In his paper, Chrisp compared the performance of the design with NURBS surfaces to designs that use conventional tilted and/or decentered rotational aspheres, and XY polynomials. The RMS Spot size over the field for different designs is shown in Fig. 10. Chrisp reported the average RMS Spot size over the field to be $61 \mu \mathrm{m}$ for the conventional aspheric design, $36 \mu \mathrm{m}$ for the XY polynomial design, and $14 \mu \mathrm{m}$ for the NURBS design. In the current design, the average RMS Spot is $8.5 \mu \mathrm{m}$, which is about $40 \%$ better comparing to the NURBS design. 


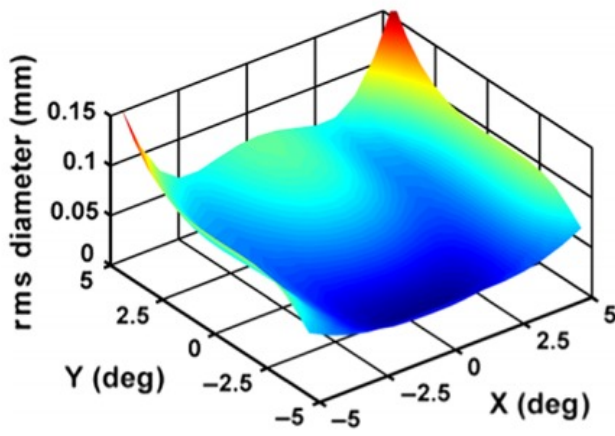

(a)

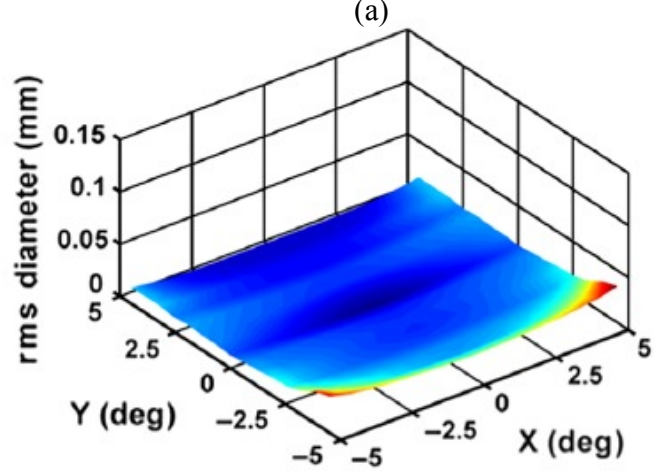

(c)

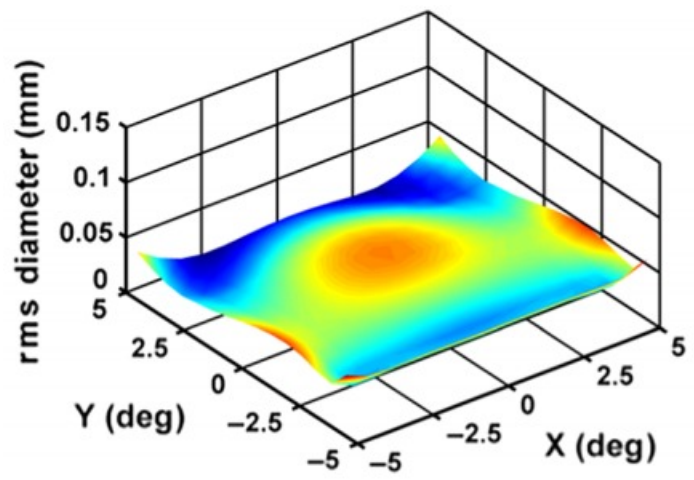

(b)

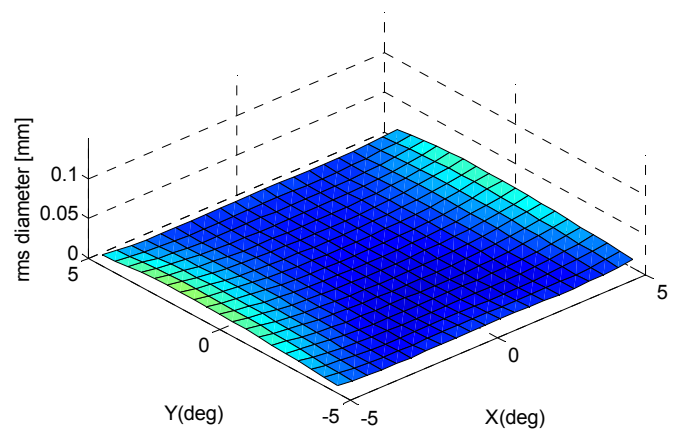

(d)

Figure 10. The RMS Spot Size over the field of the three-mirror system. (a) The design with conventional aspheric surfaces reported by Chrisp; (c) the design with XY polynomial surfaces reported by Chrisp; (c) the design with NURBS freeform surfaces reported by Chrisp; (d) the current design with aspherical surfaces $z_{f}(r)$.

Although the design presented by Crisp shows excellent performance, the surface representation with NURBS has a number of disadvantages. The major optical design programs are not capable of optimizing NURBS grid-type surfaces in imaging systems. For this reason, the optimization of the design by Chrisp was accomplished with an in-house code. Moreover, the NURBS design represents a "brute force"/" number crunching" solution, while the aspheric surface profile of the mirrors $z_{f}(r)$ is clearly able to best model the required ideal surface and to allow one to intelligently approach the optical design.

\section{MONOLITHIC FREEFORM OBJECTIVE}

So far, we have discussed the design of reflective plane-symmetric optical systems. In this section, we extend our design method to refractive optical systems and show an additional freeform surface constructed by superposition of a Cartesian oval surface and polynomial.

The Cartesian oval is an optical surface that separates two homogeneous refracting media and produces a perfect point image of a point object. In the special case of a mirror surface in which the index of refraction of object and image space media have the same magnitude but the opposite sign, the Cartesian oval solutions are conic surfaces. Other well-known solutions are a sphere for the case of aplanatic and concentric conjugate points or conic surface with the conic constant equal to the minus square of the index of refraction for the case of having one conjugate point at infinity. However, a general sag equation of the Cartesian oval is complicated. The solution for the explicit sag of the Cartesian oval has been previously discussed by other authors. Moreover, an alternate iterative method for the sag of the Cartesian oval has also been provided [10].

This iterative method solves the defining optical path length equation for the Cartesian oval for any ray from the object point $O$ to the image point $O^{\prime}$, as shown in Fig. 11 . 


$$
\begin{aligned}
& O P L_{p}-O P L_{a x i s}= \\
& \left\{n_{1} \cdot \sqrt{\left(s_{1}+S_{c}(\mathrm{r})\right)^{2}+r^{2}}+n_{2} \cdot \sqrt{\left(s_{2}-S_{c}(\mathrm{r})\right)^{2}+r^{2}}\right\}-\left\{n_{1} \cdot s_{1}+n_{2} \cdot S_{2}\right\}=0,
\end{aligned}
$$

where $S_{c}(r)$ is the sag of the Cartesian oval; $n_{l}$ and $n_{2}$ are the indexes of refraction in object and image spaces; and $s_{I}$ and $s_{2}$ are the object and image distances from the surface vertex.

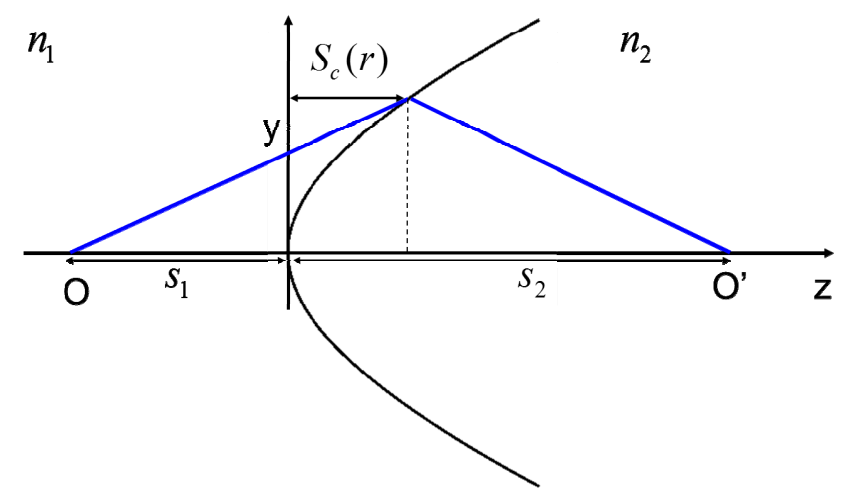

Figure 11. Geometrical variables used to define the Cartesian oval surface. All rays from the object point $O$ to the image point $O$ ' have equal optical path length.

The Cartesian oval has the property of perfectly imaging an object point into an image point with any numerical aperture. However, the imaging performance of the Cartesian oval degrades rapidly for off-axis field positions. Similarly to construction of the aspheric/freeform surface $z_{f}(r)$, the aspherical surface $z_{c}(r)$ is now constructed by adding a polynomial to the base surface $S_{c}(r)$ as,

$$
z_{c}(r)=S_{c}(r)+A_{1} r^{4}+A_{2} r^{6}+A_{2} r^{8}+\ldots
$$

where the $A$ 's represent the aspheric polynomial coefficients. A combination of surfaces $z_{f}(r)$ and $z_{c}(r)$ can be used to design certain plane-symmetric systems.

As an example, we present a monolithic freeform objective that closely resembles the monolithic freeform objective design discussed by Kiontke [11]. The design covers a vertical FOV of about $25^{\circ}$ at $\mathrm{f} / 1.4$ and operates in the long wave infrared region. The monolithic objective is made of Germanium. The initial system is constructed with confocal surfaces and comprises a freeform surface $z_{c}(r)$ to couple light from the object into the material, two freeform/aspherical surfaces $z_{f}(r)$ to bend light in the material, and additional freeform surface $z_{c}(r)$ to out-couple light towards the detector, as shown in Fig. 12. 


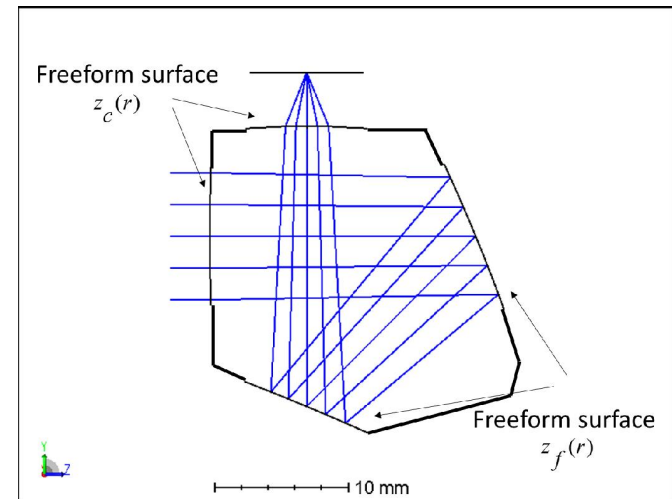

(a)

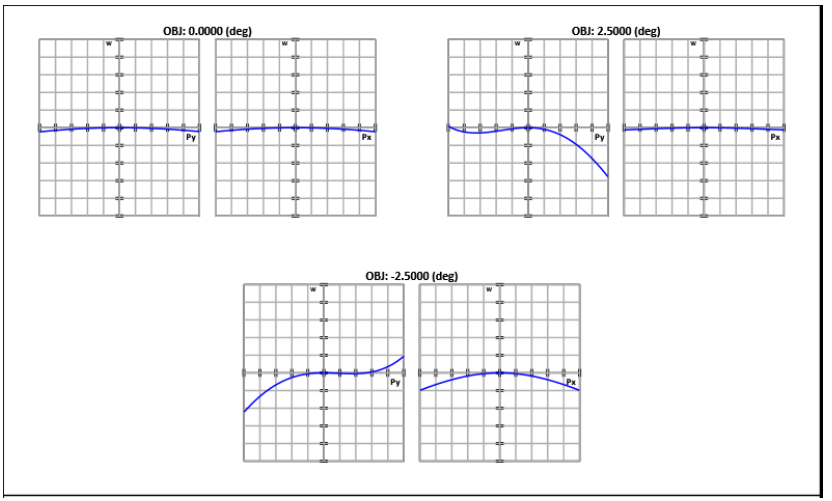

(b)

Figure 12. The starting design of a monolithic freeform objective is constructed with confocal surfaces. (a) Layout (b) OPD plots (the plot scale is 5 waves at $9 \mu m$ ). Combination of freeform surfaces $z_{f}(r)$ and $z_{c}(r)$ allows to achieve stigmatic imaging on-axis surface after surface.

Next, the Cartesian oval parameters $\left(s_{1}\right.$ and $\left.s_{2}\right)$, mirror curvatures, conic constants, and separations are removed from the optimization, and polynomial coefficients are released as variables to balance off-axis aberrations. The system layout reproduced from the paper by Kiontke and our final system layout are presented side-by-side in Fig. 13. The OPD plots and spot diagrams of our system are given in Fig. 14. Excellent, balanced performance over a FOV of $37 \times 25$ degrees has been achieved.

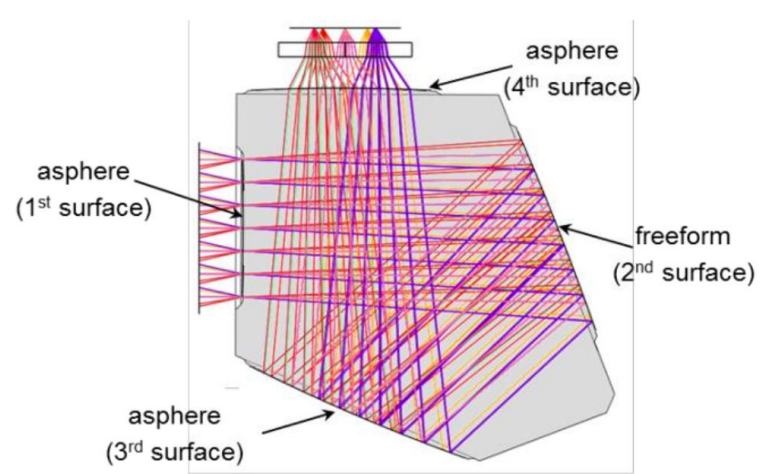

(a)

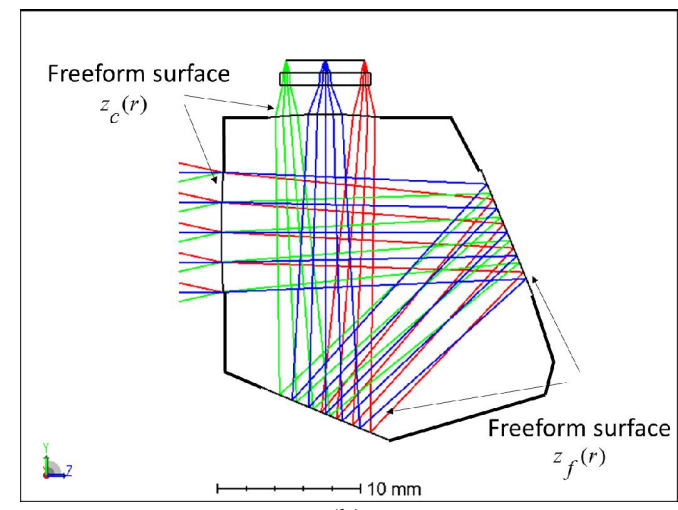

(b)

Figure 13. Layout of the monolithic objective design. (a) The design with aspheric and Zernike freeform surfaces reported by Kiontke; (b) the current design with aspherical/freeform surfaces $z_{f}(r)$ and $z_{c}(r)$. In the current design, aspheric polynomials are used to correct off-axis aberrations. 


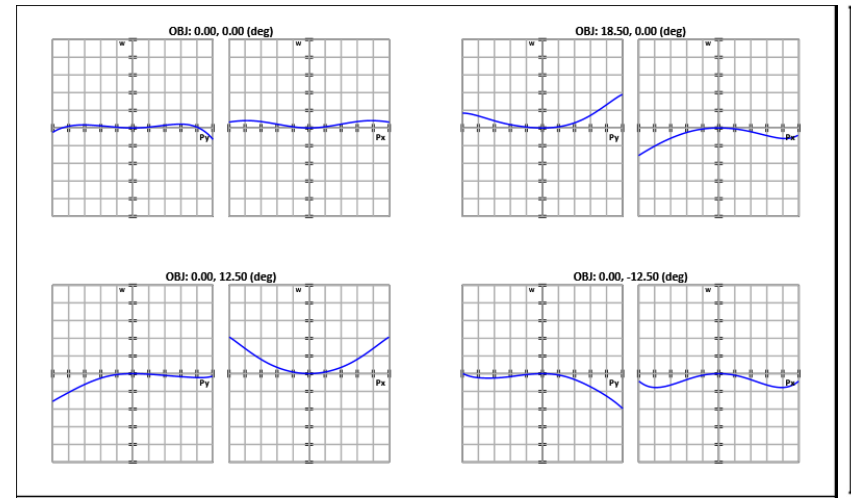

(a)

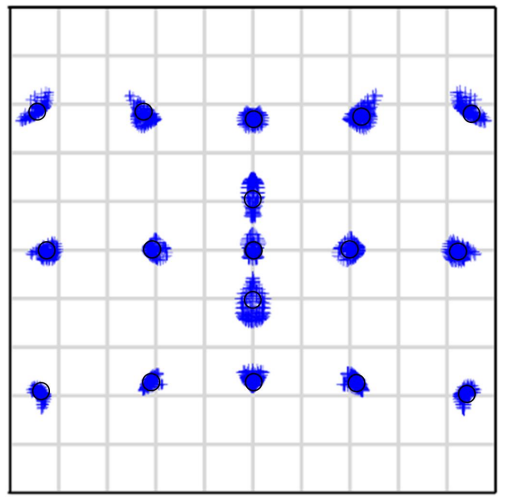

(b)

Figure 14. Imaging performance of the final monolithic objective design. (a) OPD plots (the plot scale is 1 wave at $9 \mu \mathrm{m}$ );

(b) Spot diagrams at $9 \mu \mathrm{m}$. Excellent, balanced performance over a FOV of 37x25 degrees was achieved.

The design reported by Kiontke utilizes three axially symmetric aspheric surfaces and one freeform surface. The freeform surface is described as a superposition of Zernike polynomials and is used to compensate plane-symmetric aberrations. These aspheric and freeform surface descriptions are widely accepted. However, selecting a starting point for the design, satisfying all geometrical constraints and compensating for aberrations induced by breaking up the rotational symmetry of the optical system impose challenges for the optical design. In the current design, the system geometry has already been established during the initial design step. By construction, the initial system has a reduced number of field aberrations, and only small aspheric departures to the surface sag are required to effectively correct offaxis aberrations.

\section{CONCLUSION}

To summarize, we review the methodology for the design of plane-symmetric optical systems and demonstrate an aspheric/freeform surface profile $z_{f}(r)$ constructed by superposition of a conic segment and polynomial. We also show an aspheric surface profile $z_{c}(r)$ constructed by superposition of a Cartesian oval and polynomial

The surfaces $z_{f}(r)$ and $z_{c}(r)$ are useful for designing plane-symmetric systems where the imaging is stigmatic surface after surface along the OAR. Such systems have a reduced number of field aberrations and behave closer to an axially symmetric system. Moreover, these surfaces provide additional degrees of freedom to balance aberration for the off-axis field positions and allow one to compensate for aberrations induced by breaking up the rotational symmetry of the system.

A systematic method is shown that provides a practical and effective means for establishing a starting design point and allows one to design relatively fast wide field-of-view plane-symmetric systems. We found that this specific method and corresponding aspheric/freeform surface significantly simplify the optical design task. The method have been successfully applied to design two- and three-mirror unobscured wide field-of-view reflective systems, and a monolithic freeform objective. Excellent performance over a large FOV has been achieved. The method can be extended to systems with surface tilts in two directions.

The surfaces $z_{f}(r)$ and $z_{c}(r)$ allow for optimum design or to refine the design performance of plane-symmetric optical systems by enabling a design with equivalent performance but a faster focal ratio or larger FOV than a design with convention surfaces.

\section{REFERENCES}

[1] J. M. Sasian, "How to approach the design of a bilateral symmetric optical system," Opt. Eng. 33(6) (1994).

[2] J. Sasian, "Optical design of refractive wide-field cameras," Proc. of SPIE 7060 (2008).

[3] J. M. Rogers, "Unobscured mirror design," Proc. of SPIE 4832 (2002).

[4] J. Sasian, "Design of a Schwarzschild flat-field, anastigmatic, unobscured, wide-field telescope," Opt. Eng. 29(1) (1990). 
[5] J. M. Sasian, "Review of methods for the design of unsymmetrical optical systems," Proc. of SPIE 1396 (1990).

[6] J. R. Rogers, "Techniques and tools for obtaining symmetrical performance from tilted-component systems," Opt. Eng. 39(7) (2000).

[7] J. Nelson and M. Temple-Raston, "The off-axis expansion of conic surfaces," UC TMT Report No. 91 (1982)

[8] D. Reshidko, "Topic in Modern Lens Design," PhD Dissertation, College of Optical Sciences, University of Arizona (2016)

[9] M. Chrisp, "New freeform NURBS imaging design code," Proc. SPIE 9293, 02930N (2014).

[10] C. Hsueh, T. Elazhary, M. Nakano and J. Sasian, "Closed-form sag solutions for Cartesian oval surfaces," J. Opt., Vol. 40 (4) (2011)

[11] S. R. Kiontke, "Monolithic freeform objective," Proc. of SPIE 9575 (2015) 